\title{
Sequence analysis and verification of Eimeria tenella rhomboid bait plasmid suitability for CytoTrap yeast two-hybrid system
}

\author{
Jun Zheng • Jianhua Li • Qiuyue Wang • Xiuli Xiang • \\ Pengtao Gong • Lili Cao • Yanan Cai • Guocai Zhang • \\ Xichen Zhang
}

Received: 2 June 2010 /Accepted: 16 September 2010 / Published online: 13 October 2010

(C) Springer-Verlag 2010

\section{Introduction}

Rhomboid was first described in Drosophila as a key regulator of the epidermal growth factor receptor (EGFR) signaling pathway (Wasserman et al. 2000). This function is due to its proteolytic activity towards several membranebound growth factors, which is required for their release from signal sending cells (Lee et al. 2001). The rhomboid protein family belongs to a class of serine proteases, and recent studies have also indicated that apicomplexan rhomboids have a potential role in microneme protein cleavage during host cell invasion (Dowse et al. 2005). Recent biochemical reconstitution and high-resolution crystal structures have provided proof that rhomboid proteins function as novel intramembrane proteases, with a serine protease-like catalytic apparatus embedded within the membrane bilayer, buried in a hydrophilic cavity

J. Zheng $\cdot$ J. Li $(\bowtie) \cdot$ P. Gong $\cdot$ L. Cao $\cdot$ Y. Cai $\cdot$ G. Zhang $\cdot$

$\mathrm{X}$. Zhang $(\bowtie)$

College of Animal Science and Veterinary Medicine,

Jilin University,

$5333 \mathrm{Xi}^{\circ}$ an Road,

Changchun 130062, China

e-mail: jianhuali7207@163.com

e-mail: xczhang@jlu.edu.cn

Q. Wang

Hebei Normal University of Science and Technology,

Qinhuangdao 066000, China

\section{Xiang}

Changchun Agriculture School,

Changchun 130062, China formed by a protein ring (Ha 2009). A thorough consideration of all known examples of rhomboid function suggests that despite biochemical similarity in mechanism and specificity, rhomboid proteins have different function in diverse processes (Freeman 2008).

Eimeria tenella is one of the most important and serious species causing coccidiosis in chickens (Allen and Fetterer 2002; Ding et al. 2005). By screening an E. tenella cDNA expression library with anti-sporozoite monoclonal antibodies, a new member of the rhomboid protein family was identified (Li et al. 2006). Toxoplasma gondii contains six rhomboid-like genes that we have called TgROM1TgROM6 (Timothy and Dominique 2004) to adhere to the previously proposed nomenclature for T. gondii (Sibley 1991). T. gondii rhomboid (TgROM)-like proteins containing TgROM1, TgROM2, TgROM4, and TgROM5 have recently been identified for characterization (Dowse et al. 2005; Timothy and Dominique 2004), but there is no report on function of E. tenella rhomboid (EtROM)-like protein. In this study, the rhomboid gene of $E$. tenella was analyzed and cloned into the yeast expression vector pSos, and protein was expressed in host yeast strains cdc $25 \mathrm{H}$. Then, suitability of rhomboid for CytoTrap yeast two-hybrid system was detected.

\section{Materials and methods}

General reagents and plasmid

Reverse transcriptase M-MLV (RNase $\mathrm{H}^{-}$) and restriction enzymes were purchased from TaKaRa Biotechnology 
Co., Ltd. The mouse monoclonal anti-Sos antibody and secondary antibodies for Western blotting were from BD Biosciences. Yeast two-hybrid was performed using the CytoTrap Vector Kit (Stratagene). Vectors pSos, pMyr and control plasmid pSos MAFB (positive), pMyr MAFB (positive), pSos Col 1 (negative), pMyr Lamin C (negative), and pMyr SB (positive) were provided by Stratagene.

Parasite and yeast strains

E. tenella wild strain was isolated from Xinjiang in China and was kindly provided by Dr. Jianan Li. Yeast Saccharomyces cerevisiae temperature-sensitive mutant strain $\operatorname{cdc} 25 \mathrm{H}(\alpha)$ was used that contained a point mutation at amino acid residue 1328 of the CDC25 gene. CDC25 was the yeast homologue of the Sos gene, encoding a guanyl nucleotide exchange factor that bound and activated Ras, beginning the Ras signal transduction pathway.

Construction of pSos-Rho yeast expression vector

Total RNA was isolated from freshly sporulated oocysts using Trizol ${ }^{\circledR}$ reagent (Invitrogen) according to the manufacturer's instructions. The rhomboid gene segment (GenBank accession no. DQ323509) was generated from the cDNA of E. tenella strains by PCR with primers QF (5'-TTGGTCGACGGACCTTTTGCTTCCTTCCGATC$\left.3^{\prime}\right)$ containing Sal I restriction site (underlined) and QR (5'-CGGAGCTCATGCTAATCTCGTTTTTGGCG-3') containing Sac I restriction site (underlined) and cloned into the pMD18-T vector. The $910 \mathrm{bp}$ rhomboid gene fragment was subcloned into the Sal I/Sac I sites of pSos vector. The yeast expression plasmid pSos-Rho was identified by double digestion with Sal I/Sac I and the rhomboid gene sequence was detected by Shanghai Biotechnology Co., Ltd.

Preparation of polyclonal antibodies against sporozoites of E. tenella Xinjiang strain

Sporozoites of E. tenella were first purified from sporulated oocysts as described previously (Breed et al. 1999). The purified sporozoites were frozen down in liquid nitrogen and thawed on ice for three cycles, further processed by ultrasound sonication $(300 \mathrm{~W}, 5 \mathrm{~min})$. After centrifugation at $10,000 \times \mathrm{g}$ for $15 \mathrm{~min}$, the supernatant was collected as the antigen solution for immunization. Each BALB/c mouse was injected with $0.1 \mathrm{ml}$ of antigen (equivalent to $3 \times 10^{6}$ sporozoites) emulsified with equal volume of Freund's complete adjuvant, and boost injection antigen with Freund's incomplete adjuvant after 2-week intervals. ELISA titer was measured.
Verification of yeast host strain marker phenotype

First, the temperature-sensitive phenotype of cdc25H host yeast strains was obtained from the glycerol stock by scraping off splinters of solid ice with a sterile wire loop or sterile inoculating stick, and then the splinters were streaked onto a YPAD agar plate. At the same time, yeast was streaked from the $-80^{\circ} \mathrm{C}$ glycerol stock onto each of the four agar "dropout" plates (Four sets of SD agar plates were prepared using the appropriate $10 \times$ dropout solutions to test the $\operatorname{cdc} 25 \mathrm{H}$ yeast strain for the following nutritional requirements: tryptophan, leucine, histidine, and uracil). The plates were incubated at room temperature $\left(22-25^{\circ} \mathrm{C}\right)$ for 4-6 days. After the phenotype had been verified, colonies from the YPAD plate were used to inoculate medium for the preparation of competent yeast cells.

Preparation of cdc25H yeast competent cells and transformation of bait plasmid

The yeast competent cells were perpetrated as described previously (Gietz et al. 1995), using the CytoTrap yeast two-hybrid system (Stratagene). Preboiled sheared salmonsperm DNA $(20 \mathrm{mg} / \mathrm{ml})$ was added to each transformed tube together with the recombinant rhomboid bait plasmid (pSos-Rho) and control plasmid pSos to be transformed (400 ng). Competent cells $(50 \mu \mathrm{l})$ were added to the DNA mixture followed by addition of $1.2 \mathrm{ml} \mathrm{PEG/LiAc} \mathrm{solution}$ (40\% PEG3350 in $100 \mathrm{mM} \mathrm{LiAc/TE).} \mathrm{The} \mathrm{DNA-cell}$ mixture was incubated for $30 \mathrm{~min}$ at $25^{\circ} \mathrm{C}$ on a rotator. DMSO was added $(100 \mu \mathrm{l})$ to the transformation mix and incubated at $42^{\circ} \mathrm{C}$ for $10 \mathrm{~min}$. Cells were centrifuged for $1 \mathrm{~min}$ in an Eppendorf centrifuge. The supernatant was discarded, and the cell pellet was resuspended in $1 \mathrm{M}$ sorbitol $(150 \mu \mathrm{l})$ and plated on the SD/glucose $(-\mathrm{L})$ plates incubated at $24-25^{\circ} \mathrm{C}$ for $4-6$ days.

Purification and Western analysis of recombinant rhomboid bait protein

One colony was picked from each $\mathrm{SD} /$ glucose $(-\mathrm{L})$ plates (yeast cells transformed pSos-Rho and pSos), then inoculated in $10 \mathrm{ml} \mathrm{SD} /$ glucose $(-\mathrm{L})$ liquid medium which was performed on a $250-$ rpm rotator at $24-25^{\circ} \mathrm{C}(2-3$ days, until $\left.\mathrm{OD}_{600}>1.0\right)$. The yeast cells were pelleted by spinning the culture at $1,000 \times \mathrm{g}$ for $5 \mathrm{~min}$ at room temperature. Then, the yeast cell pellet was resuspended in $200 \mu$ of cell lysis buffer for protein isolation containing freshly added protease inhibitors $(1 \mathrm{mM}$ PMSF, $10 \mu \mathrm{g} / \mathrm{ml}$ aprotinin, $1 \mu \mathrm{M}$ pepstatin $\mathrm{A}, 100 \mu \mathrm{M}$ leupeptin, and $1 \mu \mathrm{g} / \mathrm{ml}$ chymostatin). The cells were vortexed for $5 \mathrm{~min}$ at $4^{\circ} \mathrm{C}$ with an equal volume of acid-washed glass beads $(0.5 \mathrm{~mm}$ in diameter, Stratagene). The cell lysis was monitored by 
phase-contrast microscopy until $>70 \%$ of the yeast cells appear transparent. The lysate was collected by centrifugation at $12,000 \times \mathrm{g}$ for $5 \mathrm{~min}$ at $4^{\circ} \mathrm{C}$. The lysate was verified by sodium dodecyl sulfate polyacrylamide gel electrophoresis (SDS-PAGE) and Western blot analysis using the mouse monoclonal anti-Sos antibody (BD Biosciences) and polyclonal antibodies that immunoreacted with the sporozoites of E. tenella.

Indirect immuno-fluorescence assay (IFA)

All manipulations were carried out at room temperature. The yeast cells transformed with plasmid pSos-Rho in centrifuge tube was fixed with $4 \%$ paraformaldehyde for $20 \mathrm{~min}$. Following fixation, cells were rinsed in PBS$0.1 \mathrm{M}$ glycine, then permeabilized in PBS, $0.2 \%$ Triton-X100 for $20 \mathrm{~min}$ and blocked in the same buffer with $2 \%$ BSA. The tube was incubated for 60 min with polyclonal antibodies diluted in blocking solution, washed and incubated for $60 \mathrm{~min}$ with goat anti-mouse IgG-FITC antibody, diluted 1:2,000 in blocking solution. Yeast cells diluted with PBS on glass slides were mounted in vectashield and kept at $4^{\circ} \mathrm{C}$ in the dark. Fluorescent images were examined under a Nikon microscope. Adobe Photoshop (Adobe Systems, Mountain View, CA) was used for image processing.

Verification of $E$. tenella rhomboid bait plasmid suitability

Four groups of plasmid were co-transformed into the yeast host for the following verification: plasmid pSosMAFB (400 ng) and pMyrMAFB (400 ng) were co-transformed as positive control group; pSosMAFB (400 ng) and pMyr laminC (400 ng) pair served as negative controls; pSos-Rho (400 ng) and pMyr (400 ng) were co-transformed to detect suitability of the bait protein; and pSos-Rho (400 ng) and pMyrSB (400 ng) pair served as a control that confirms the integrity of the pSos vector. Each transformation mixture was spread on $\mathrm{SD} /$ glucose (-UL) agar plates by dropping approximately ten non-acid-washed glass beads until the mixture was spread on the surface. Once the plate was dry, the glass beads were poured off, and these plates were incubated at room temperature $\left(22-25^{\circ} \mathrm{C}\right)$ until colonies were visible (4-6 days). Then, three colonies were picked and resuspended in the $25 \mu \mathrm{l}$ sterile $\mathrm{H}_{2} \mathrm{O}$, respectively; $2.5 \mu \mathrm{l}$ of the yeast $/ \mathrm{H}_{2} \mathrm{O}$ suspensions was spotted onto each of two SD/galactose (-UL) agar plates and two SD/ glucose (-UL) agar plates. One plate of each type was incubated at $37^{\circ} \mathrm{C}$. The second plate of each type was kept at room temperature $\left(22-25^{\circ} \mathrm{C}\right)$ for 5 days. The $\mathrm{SD} /$ galactose (-UL) agar plates that were incubated at $37^{\circ} \mathrm{C}$ required up to 7-10 days of incubation time for yeast colonies to appear.

\section{Results}

Sequence analysis of E. tenella rhomboid-like protein

Here, the phylogenetic tree of amino acid sequence of rhomboid-like protein that named EtROMz from E. tenella and other rhomboid-like proteins (ROMs) from $T$. gondii, $P$. falciparum, and Drosophila melanogaster is shown in Fig. 1a. The highest homology was between EtROMz and TgROM3 according to the amino acid sequence comparison. Rhomboids are conserved in apicomplexan parasites, some being of similar size to Drosophila rhomboid-1 and others being significantly larger. EtROMz is included in smaller ROMs. The amino acid sequence of alignment of EtROMz, TgROM3, and PfROM3 is shown in Fig. 1b. All sequences contain TM domains as predicted by TMHMM (http://www.cbs.dtu.dk/services/TMHMM-2.0/). More conserved or identical residues are found in TM domains between EtROMz and other ROM3. The apicomplexan ROMs contain many of the critical conserved residues shown to be required for Drosophila rhomboid-1 activity. Sinisa Urban (Urban et al. 2001) demonstrated that mutations of $\mathrm{W}, \mathrm{R}, \mathrm{N}, \mathrm{G}, \mathrm{S}$ (the catalytic serine), and $\mathrm{H}$ abolished rhomboid activity. EtROMz residues corresponding to these are conserved in all apicomplexan ROMs. Moreover, every basic group of EtROMz cDNA sequence can be found in Contig7050 which was obtained from the Eimeria Tenella Genome Project using the 27 July 2004 assembly except the last $11 \mathrm{bp}$. We suppose that EtROMz is rhomboid 3 in E. tenella. This presumption is conformed with report of Dowse and Soldati (Dowse and Soldati 2005).

\section{Construction of pSos-Rho yeast expression vector}

The rhomboid gene was cloned into pMD18-T vector and yeast expression vector pSos (pSos-Rho). Restriction endonuclease Sal I/Sac I digestion patterns showed that there was a 910-bp purpose fragment, and it was inserted correctly into the vector (Fig. 2). The rhomboid gene sequence was detected by Shanghai Biotechnology Co., Ltd, and it was conformed to rhomboid-like gene sequence of E. tenella in gene bank (DQ323509).

Yeast host strain marker phenotype

Yeast colony streaked on four SD agar plates (SD/glucose (-Try), SD/glucose (-Leu), SD/glucose (-His), and SD/ glucose (-Ura)) was not emergent until incubated at $25^{\circ} \mathrm{C}$ on the sixth days, but there are much colony on YPAD plate (Table 1). The genotype of $\operatorname{cdc} 25 \mathrm{H}$ yeast strain $(\alpha)$ was MAT $\alpha$ ura3-52 his3-200 ade2-101 lys2-801 trp1-901 leu23112 cdc25-2 Gal+, so the phenotype of yeast strain has 
a

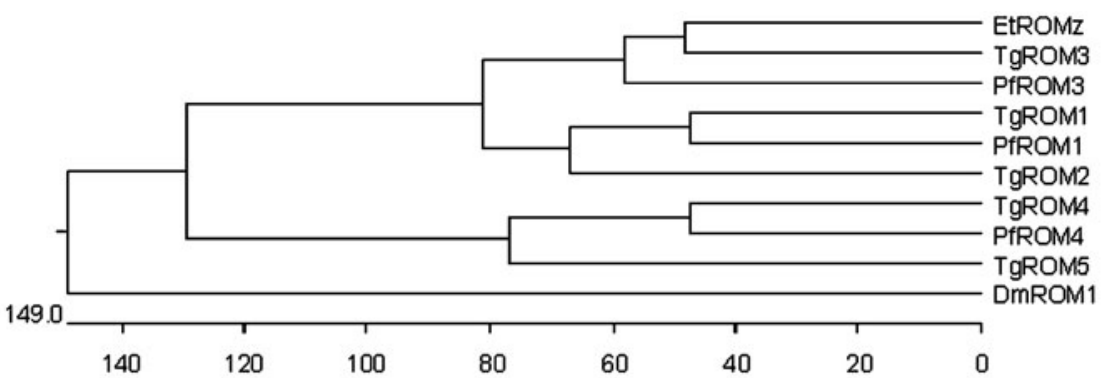

b

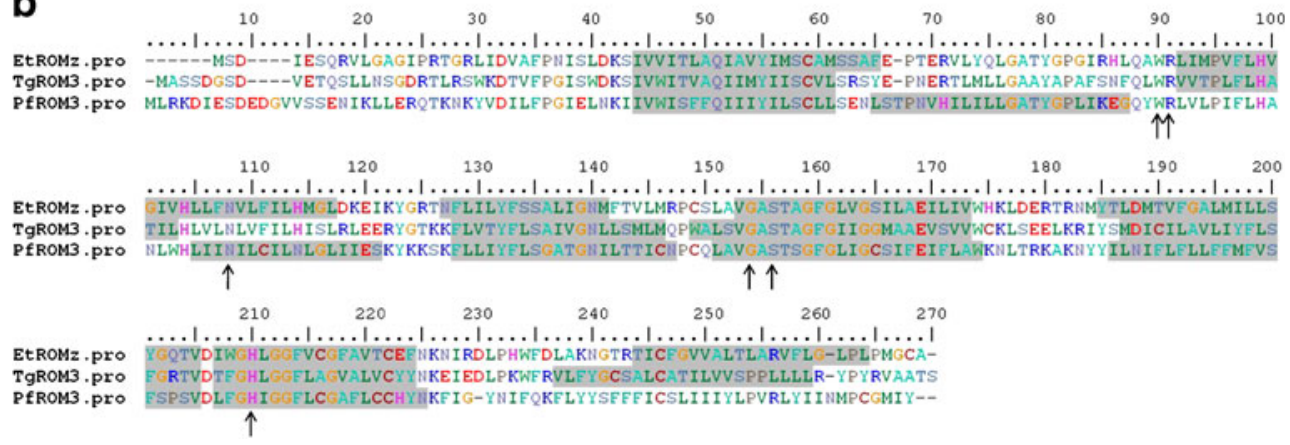

Fig. 1 Sequence analysis of EtROMz sequences. a Phylogenetic tree of the ROMs from some apicomplexan species. Species abbreviations: Dm, D. melanogaster; Et, E. tenella; Pf, Plasmodium falciparum; Tg, T. gondii. GenBank accession numbers are as follows: EtROMz, ABC50099; TgROM1, AAT29065; TgROM2, AAT29066; TgROM3, AAT39987; TgROM4, AAT29067; TgROM5, AAT47708; PfROM1, PF11_0150; PfROM3, MAL8P1.16; PfROM4, PFE0340c; and
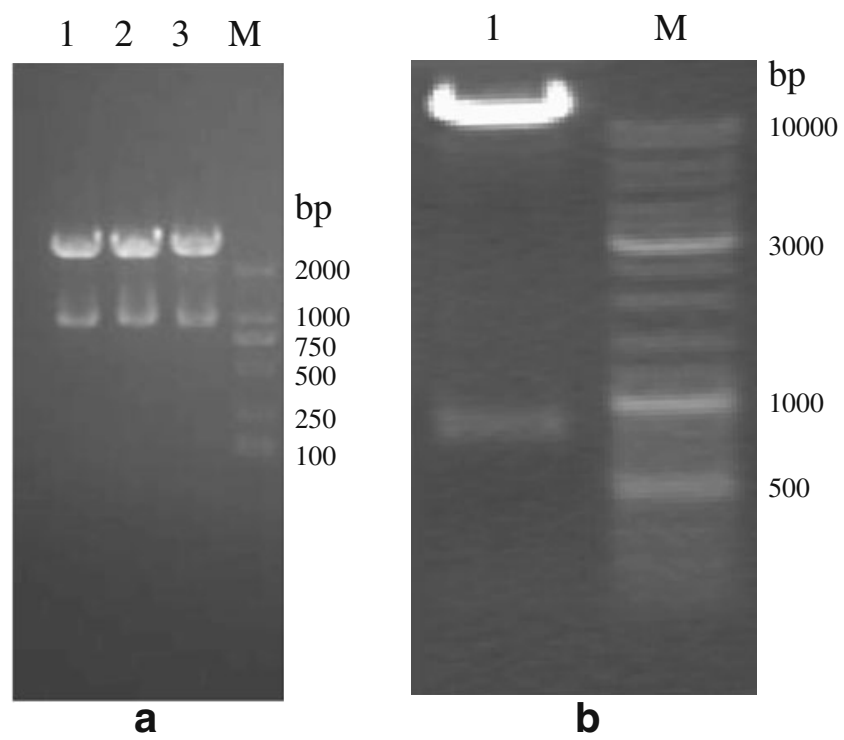

Fig. 2 Restriction endonuclease Sal I/Sac I digestion patterns. a Characterization of pMD18-T-Rho by restriction enzyme digestion and analyzed by electrophoresis. $M$, DNA Marker 2000; 1, 2, 3, pMD18-T-Rho digested by Sal I/Sac I. b Characterization of pSosRho by restriction enzyme digestion and analyzed by electrophoresis. $M$, DNA Marker 10000; 1 , pSos-Rho digested by SalI/SacI
DmROM1: AAF47496. b Alignment of the TM domains and some surrounding amino acids of EtROMz, TgROM3, and PfROM3 from the E. tenella, T. gondii, and P. falciparum of Apicomplexa. The TM domains predicted by TMHMM (http://www.cbs.dtu.dk/services/ TMHMM-2.0/) are shown in gray. Residues corresponding to $\mathrm{W}, \mathrm{R}$, $\mathrm{N}, \mathrm{G}, \mathrm{S}$, and $\mathrm{H}$ of ROMs are marked with arrows

been verified (growth on the YPAD plate and no growth on any of the four SD agar dropout plates), and the colonies from the YPAD plate could be used to prepare competent yeast cells.

Verification of bait protein by SDS-PAGE and Western blotting

In order to examine expression of recombinant protein in yeast cells, cdc $25 \mathrm{H}$ yeast cells were transformed with pSos and pSos-Rho plasmid. The protein extracts were then analyzed by SDS-PAGE and Western blotting (Fig. 3). In Fig. 3a, two proteins banded with the molecular weight of 170 and $198 \mathrm{kDa}$ were detected, showing that the hSos protein was expressed by pSos and the hSos-rhomboid fusion protein was expressed by pSos-Rho plasmid. In Fig. 3b, two bands at about 170 and $198 \mathrm{kDa}$ were recognized by mouse anti-Sos monoclonal antibody in protein extracts of yeast cells transfected with pSos and pSos-Rho plasmid (Fig. 3c, lane 1 and 2). In Fig. 3c, a band at about $198 \mathrm{kDa}$ was recognized by mouse antisporozoites of E. tenella polyclonal sera in protein extracts of cells transfected with pSos-Rho plasmid (Fig. 3c, lane 2). The mouse anti-sporozoites of E. tenella polyclonal sera antibody did not react with lysate of yeast cells transfected 
Table 1 Test on the phenotype ability of $\operatorname{cdc} 25 \mathrm{H}(\alpha)$

\begin{tabular}{lllll}
\hline & SD/glucose (-Trp) & SD/glucose (-Leu) & SD/glucose (-His) & SD/glucose (-Ura) \\
\hline $\operatorname{cdc} 25 \mathrm{H} \alpha$ & - & - & - & - \\
\hline
\end{tabular}

+ represents the cells grow well, - represents the cells fail to grow

with pSos plasmid (Fig. 3c, lane 1). These results indicated that the Sos-rhomboid fusion protein was expressed by pSos-Rho plasmid.

Indirect immuno-fluorescence assay

Yeast cells were transformed with the recombinant plasmid pSos-Rho and pSos. The expression of the fusion proteins were detected by immuno-fluorescence staining with mouse anti-sporozoites of E. tenella serum and goat anti-mouse IgG-FITC antibody. The green fluorescence was observed under a laser scanning confocal microscope for cells transformed with the recombinant plasmid pSos-Rho (Fig. 4), not for cells transformed with pSos vector. Furthermore, the green fluorescence indicated that SosRho fusion protein accumulated in plasma membrane region of the yeast cells (Fig. 4a). The results suggested that EtROM was possibly expressed to approach yeast plasma membrane.

Suitability of rhomboid for CytoTrap interaction assays

White madid patch colonies grew at room temperature on $\mathrm{SD} /$ glucose (-UL) fresh plates containing four groups until the fifth day. Six days later, the candidates producing patches didn't grow on $\mathrm{SD} /$ glucose (-UL) plates at $37^{\circ} \mathrm{C}$.
The negative control group co-transforming pSosMAFB and pMyr laminC did not grow on SD/galactose (-UL) plates, but the patches could be detected for other groups (Table 2). This result indicated that recombinant bait protein has integrity and didn't have virulence for host strain, but hSos protein was located to the plasma membrane, so the Ras-signaling pathway was activated and the cdc $25 \mathrm{H}$ yeast strain was allowed to grow at $37^{\circ} \mathrm{C}$.

\section{Discussion}

We reported the characterization of members of the rhomboid family of proteases in the parasite E. tenella. The rhomboid gene was discovered from a systematic analysis of EGFR signaling in Drosophila, where it encodes a seven-trans-membrane protein (Urban et al. 2001; Xiao et al. 1996; Herra et al. 2010). Although rhomboids throughout evolution are diverse, the overall structure of several TM domains is conserved (Urban and Shi 2008). Recent high-resolution crystal structures of rhomboid have provided proof that rhomboid proteins function as novel intramembrane proteases, with a serine protease-like catalytic apparatus embedded within the membrane bilayer, buried in a hydrophilic cavity formed by a protein ring. An agreement for the nomenclature of

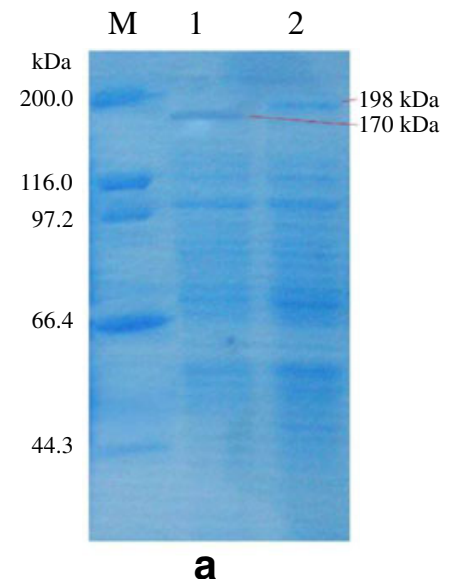

Fig. 3 Detection of rhomboid protein expression. a The Sos and SosRho fusion protein were expressed in yeast cells and evaluated by SDS-PAGE and stained with Coomassie blue. $M$, protein MW marker (high); lane 1, Sos protein expressed by pSos plasmid; 2, Sos-Rho fusion protein expressed by pSos-Rho. b Western blot of yeast cell lysates using anti-Sos monoclonal antibody to detect Sos and Sos-Rho fusion protein expression. c Western blot of yeast cell lysates using mouse anti-sporozoites of E. tenella polyclonal sera to detect rhomboid protein expression. All size markers denote kilodalton 


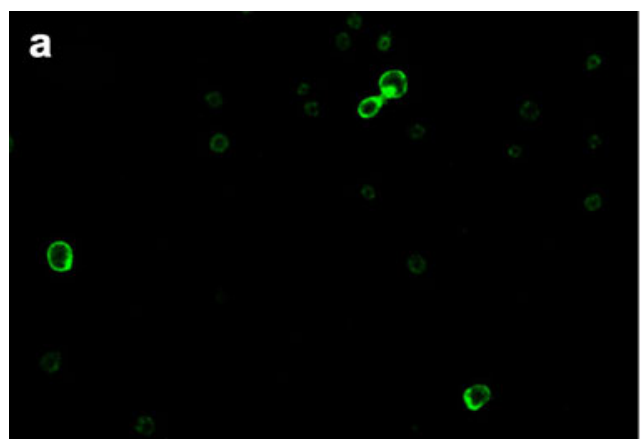

Fig. 4 Indirect IFA of rhomboid protein in yeast cells. a Yeast cells were transformed with recombinant plasmid pSos-Rho. b Yeast cells were transformed with plasmid pSos. The yeast cells were fixed, and

apicomplexan rhomboids has been made between our groups and others (Dowse and Soldati 2005). Six genes coding for rhomboid-like proteins (ROMs) have been found in $T$. gondii, called TgROM1-6. The numbering of $T$. gondii ROMs was applied in the order in which they were identified. The six genes identified in $T$. gondii code for three smaller rhomboids, two larger rhomboids, and one putative mitochondrial rhomboid, but in E. tenella, so far only one ROM (accession no. DQ323509) has been isolated from a lambda cDNA library with a monoclonal antibody against sporozoite ( $\mathrm{Li}$ et al. 2006).

Amino acid sequence of EtROMz was aligned with rhomboid of other apicomplexan parasites; more nearly, genetic relationship has been searched between EtROMz and TgROM3 because of significantly resemblance of their transmembrane domain (TMD) amino acid sequence. Then, EtROMz nucleotide sequence was contrasted with Contig7050 of E. tenella genome which was reported by Dowse and Soldati. We found that all ROMz nucleotide sequence was found out in Contig7050. Therefore, ROMz was possibly ROM3 in E. tenella. This deduction was conformed with the Dowse and Soldati report that Contig7050 was ROM3 in E. tenella.

Sos recruitment system was first preferred in 1997 (Aronheim et al. 1997) and then Broder devised Ras recruitment system (Broder et al. 1998). The CytoTrap system is based upon the ability of the Sos protein, to complement the $c d c 25$ defect and the generating fusion proteins whose interaction in the yeast cytoplasm activates

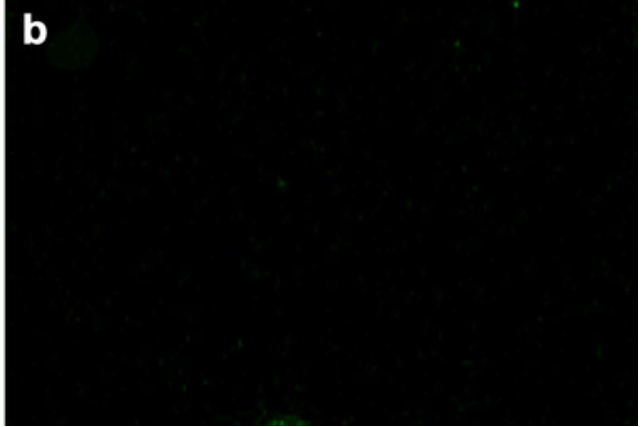

IFAs were performed using an immune serum against sporozoites of E. tenella followed by goat anti-mouse IgG-FITC antibody. Fluorescent images were examined

the Ras-signaling pathway, inducing cell growth (Barak et al. 2001). These properties of the CytoTrap system enable the study of protein interactions that cannot be assayed by conventional two-hybrid or interaction trap systems. These include proteins that are transcriptional activators or repressors, proteins that require post-translational modification in the cytoplasm, and proteins that are toxic to yeast in conventional two-hybrid systems (Jun et al. 2009).

The signaling function of rhomboid proteins was believed to be conserved in eukaryotes and prokaryotes (Urban et al. 2002). The proteins encoded by ETRH01 were likely to share common functions with other well-studied organisms of rhomboid proteins, especially with rhomboids of $T$. gondii. Experimental data from $T$. gondii have linked rhomboid protein with the invasion process (Kami 2004). Recent studies have also indicated that a spatially localized rhomboid protease cleaves cell surface adhesins that are essential for invasion by Toxoplasma (Brossier et al. 2005). As for the ETROMz encoded protein, interacted protein with ROMz was tried to screen by CytoTrap yeast twohybrid system, but when recombinated bait plasmid was transformed in yeast host strain, the Ras system of cdc $25 \mathrm{H}$ host strain was activated, accordingly, host strain could grow at $37^{\circ} \mathrm{C}$. The experimental result indicated that bait fusion protein was completely located on yeast cellular membrane, so the hSos protein was near to the cellular membrane and Ras system was activated. This result demonstrated that EtROMz protein was expressed in cellular membrane of $\operatorname{cdc} 25 \mathrm{H}$ yeast host strain. Although
Table 2 Test on the selfactivation and fixing ability of the bait vector

\footnotetext{
+ represents the cells grow well,

- represents the cells fail to grow
}

\begin{tabular}{|c|c|c|c|c|}
\hline \multirow[t]{2}{*}{ Group } & \multirow[t]{2}{*}{ Plasmids transformed } & \multirow{2}{*}{$\begin{array}{l}\mathrm{SD} /(-\mathrm{UL}) 25^{\circ} \mathrm{C} \\
\text { Glucose }\end{array}$} & \multicolumn{2}{|c|}{$\mathrm{SD} /(-\mathrm{UL}) 37^{\circ} \mathrm{C}$} \\
\hline & & & \multicolumn{2}{|c|}{ Glucose galactose } \\
\hline 1 & pSosMAFB + pMyrMAFB & + & - & + \\
\hline 2 & pSosMAFB + pMyr laminC & + & - & - \\
\hline 3 & pSos-Rho+pMyr & + & - & + \\
\hline 4 & pSos-Rho+pMyrSB & + & - & + \\
\hline
\end{tabular}


there were seven TMDs in rhomboid in most apicomplexan parasites, the expressed location was different in every being, and there was no report about expression in yeast cell for rhomboid protein of parasite.

Acknowledgements This work was supported by grants from the Foundation of Jilin Provincial Science and Technology Department of China (20070135) and the National Natural Science Foundation of China (nos. 30170696, 30500370, and 30671580).

\section{References}

Allen PC, Fetterer RH (2002) Recent advances in biology and immunobiology of Eimeria species and in diagnosis and control of infection with these coccidian parasites of poultry. Clin Microbiol Rev 15:58-65

Aronheim A, Zandi E, Hennernann $\mathrm{H}$ et al (1997) Isolation of an AP-1 repressor by a novel method for detecting protein-protein interactions. Mol Cell Biol 17:3094-3099

Barak, Huang W, Wang SL et al (2001) cDNA library screening using the SOS recruitment system. Biotechniques 30(1):94-99

Breed DG, Schetters TP, Verhoeven NA, Boot-Groenink A, Dorrestein J, Vermeulen AN (1999) Vaccination against Eimeria tenella infection using a fraction of $E$. tenella sporozoites selected by the capacity to activate T cells. Int J Parasitol 29:1231-1240

Broder YC, Katz S, Aronheim A (1998) The Ras recruitment system, a novel approach to the study of protein-protein interactions. Curr Biol 8:1121-1124

Brossier F, Jewett TJ, Sibley LD, Urban S (2005) A spatially localized rhomboid protease cleaves cell surface adhesins essential for invasion by Toxoplasma. Proc Natl Acad Sci USA 102:4146-4151

Ding X, Lillehoj HS, Dalloul RA, Min W, Sato T, Yasuda A, Lillehoj EP (2005) In ovo vaccination with the Eimeria tenella EtMIC2 gene induces protective immunity against coccidiosis. Vaccine 23:3733-3740

Dowse TJ, Soldati D (2005) Rhomboid-like proteins in Apicomplexa: phylogeny and nomenclature. Trends Parasitol 21:254-258

Dowse TJ, Pascall JC, Brown KD, Soldati D (2005) Apicomplexan rhomboids have a potential role in microneme protein cleavage during host cell invasion. Int J Parasitol 35:747-756
Freeman M (2008) Rhomboids: seven years of a new protease family. Semin Cell Dev Biol 10:1-32

Gietz RD, Schiestl RH, Williems AR et al (1995) Studies on the transformation of intact yeast cells by the LiAc/SS-DNA/PEG procedure. Yeast 11(4):355-360

Ha Y (2009) Structure and mechanism of intramembrane protease. Semin Cell Dev Biol 20:240-250

Herra A, Mckenzie L, Suryadinata R, Sadowski M, Parsons LM, Sarcevic B, Richardson HE (2010) Geminin and Brahma act antagonistically to regulate EGFR-Ras-MAPK signaling in Drosophila. Dev Biol 2:7-9

Jun Chen, Jianhong Zhou, Sanders Claire K, Nolan John P, Hong Cai (2009) A surface display yeast two-hybrid screening system for high-throughput protein interactome mapping. Anal Biochem 390(1):29-37

Kami Kim (2004) Role of proteases in host cell invasion by Toxoplasma gondii and other Apicomplexa. Acta Trop 91(1):69-81

Lee JR, Urban S, Garvey CF, Freeman M (2001) Regulated intracellular ligand transport and proteolysis control EGF signal activation in Drosophila. Cell 107:161-171

Li J, Zhang X, Liu Q, Yin J, Yang J (2006) Eimeria tenella: cloning of a novel Eimeria tenella cDNA encoding a protein related to rhomboid family from F2 hybrid strain. Exp Parasitol 113:215-220

Sibley LD, Pfefferkornb ER, Boothroyd JC (1991) Proposal for a uniform genetic nomenclature in Toxoplasma gondii. Parasitol Today 7:327-328

Timothy Dowse, Dominique Soldati (2004) Host cell invasion by the apicomplexans: the significance of microneme protein proteolysis. Curr Opin Microbiol 7(4):388-396

Urban S, Shi Y (2008) Core principles of intramembrane proteolysis: comparison of rhomboid and site-2 family proteases. Curr Opin Struct Biol 18:432-441

Urban S, Leea JR, Freeman M (2001) Drosophila rhomboid-1 defines a family of putative intramembrane serine proteases. Cell 107:173-182

Urban S, Leea JR, Freeman M (2002) A family of Rhomboid intramembrane proteases activates all Drosophila membranetethered EGF ligands. EMBO J 21:4277-4286

Wasserman JD, Urban S, Freeman M (2000) A family of rhomboid-like genes: Drosophila rhomboid-1 and roughoid/rhomboid-3 cooperate to activate EGF receptor signaling. Genes 14:1651-1663

Xiao H, Hrdlicka LA, Nambu JR (1996) Alternate functions of the single-minded and rhomboid genes in development of the Drosophila ventral neuroectoderm. Mech Dev 58:65-74 